\title{
Book Review: Researching Student Learning in Higher Education: A Social Realist Approach
}

\author{
By Jennifer M. Case \\ London: Routledge, 2013. 168 pp. \\ ISBN: 978-0-415-66234-5
}

Margaret Archer, a renowned British sociologist and social realist, argues that the distinction between structure and agency is most readily observed in the realm of culture. Structure, as the domain of social posturing, has to do with production, while agency, as the domain of human action, has to do with consumption. Instead of ascribing causal primacy to actions in social settings to either structure (social dependency) or agency (self-sufficiency), Archer contends that it is more important to consider the cultural contexts in which the settings have developed and the actions have occurred.

In the context of higher education, Jennifer M. Case suggests that it is impossible to focus on teaching and learning without examining the social milieu in which these interactions are taking place. In Researching Student Learning in Higher Education: A Social Realist Approach, a theoretical study of student access and achievement, Case appropriates Archer's constructions of structure and agency to usurp the simple input-output model of higher education in favor of a more culturally sensitive approach. Working sociologically, she moves towards a realist understanding of "what changes in student agency might be considered desirable outcomes for higher education" and "how the system will need to change (structurally and culturally) to better enable the kinds of changes in student agency that are desired" (12).

The first part of the book creates an international and historical context for examining contemporary challenges and researching student learning in higher education. Case, as a professor in the Department of Chemical Engineering at the University of Cape Town, South Africa, brings her own perspective to the table; however, she does so in order to illustrate why a theory that allows for an exchange between structure and agency might be needed, rather than to advance a particular discipline or agenda. Her contention here is that "a sophisticated sociological approach will be needed in order to do justice to the demands for a nuanced and productive line of research" (15).

The second part of the book, which focuses on the underpinnings of Archer's theory, presents an overview of social realism and describes its applications to higher education. For Case, "a realist perspective on education and society offers great promise towards building a deeper understanding of the social dynamics that underpin observed outcomes in education" (35). In one chapter, she discusses the ontological and epistemological foundations of Roy Bhaskar's critical realism, a theory for revealing the "invisible apparatuses" that inform the realm of human experience. In the next chapter, she extrapolates on her initial construction of social realism, especially on Archer's use of morphogenesis (change) and morphostasis (stagnancy). And in the last two chapters, she explains Basil Bernstein's realist perspective on knowledge and curriculum, and shows how all three theories-Archer's, Bhaskar's, and Bernstein's - can be used to develop a theory of student success.

The third part of the book, and perhaps the most substantial, is dedicated to developing a case study in engineering education at the University of Cape Town. The first two chapters describe the geographical and disciplinary contexts of engineering education, especially in South Africa, while the balance explores what it might take to change the system in 
order to encourage a broader range of student participation and success. The narratives in this section, which suggest that peer support, including social networking, is a key enabler of student success, are particularly useful for demonstrating how a social realist approach can be used to examine student learning outcomes. "[A] deep approach to learning, which prioritizes conceptual understanding, has been underscored in this study," Case writes. "However, we also see other actions that are necessary to achieve academic success...in what students themselves report as being important" (124). Based on her analysis, Case makes several recommendations for expanding student participation, including the need to ensure a rigorous curriculum and the need to be receptive to change.

Finally, in the fourth part of the book, Case pulls the strands together to show how Archer's theory can produce a stratified model of human agency in the context of higher education: "here we can see that personal identity and social identity are distinct entities, but in tracking their interplay we can note the possibilities for full agential morphogenesis in the student: the emergence of the social actor, who is able to invest him or herself in a social role that expresses what they care about in the world" (137). Her argument, simply put, is that any explanation of student agency that does not account for the culturally-constructed nature of knowledge will be limited.

The strength of Researching Student Learning in Higher Education: A Social Realist Approach lies in Case's application of social realist theory to a case study in engineering education. Through a series of student narratives, she unpacks some of Archer's more difficult concepts, such as structural conditioning (how a society constrains or enables its members) and situational logics (the compliments and contradictions of culture and society), and demonstrates how a culturally-competent model of higher education could be replicated across contexts to improve student access and achievement. Case admits that the current space for the morphogenesis of student agency is limited, especially among marginalized populations; however, this does not detract from her argument that there is space for improvement.
This book is useful for those who wish to explore options outside of the traditional input-output model of higher education. It is also suited for those with an interest in social realist theory and its application to the teaching and learning process, especially in the context of engineering education. As more students gain access to higher education, it is important to have measures, such as the social realist model, in place to meaningfully describe their experiences. Rationalist models that ignore the contributions of disenfranchised players, including women and Third World professionals, are no longer sufficient, and Case's book is a good place to start looking for alternatives.

Reviewed by Daniel Casebeer University of Pittsburgh, USA 